\title{
Inhalts verzeichnis
}

Erstes Kapitel

Die Arbeitsgegenstände im System der gesellschaftlichen Produktion . . 11

1. Arbeitsgegenstände und Technik . . . . . . . . . . . . . . . . . 11

2. Arbeitsgegenstände und wissenschaftlich-technischer Fortschritt . . . 24

3. Materialintensität und Nutzeffekt der Produktion . . . . . . . . 36

4. Faktoren ůd Kennzifferı der Materialintensität . . . . . . . . . . 44

\section{Zqeites Kapitel}

Die wachsende Bedeutung neuer Werkstoffe in der Wirtschaft der industriell entwickelten Länder . . . . . . . . . . . . . . . . . . . .

1. Die objektiven Voraussetzungen zur Schaffung neuer Werkstoffe und das Wesen der Kategorie „neue Werkstoffe“ . . . . . . . . . . . .

2. Die Bedeutung neuer Werkstoffe für Technik und Alltag. . . . . . .

3. Die Rolle der Wissenschaft bei der Entwicklung und Verbesserung der Eigenschaften neuer Werkstoffe . . . . . . . . . . . . 72

4. Die Klassifikation der neuen Werkstoffe . . . . . . . . . . 78

Drittes Kapitel

Methodische Fragen der Bestimmung der volkswirtschaftlichen Effektivität neuer Werkstoffe . . . . . . . . . . . . . . . . . . 87

1. Einige allgemeine Probleme . . . . . . . . . . . . . 87

2. Prinzipien zur Effektivitätsbestimmung von Einsatzvarianten neuer Werkstoffe .................... 101

3. Methoden der Effektivitätsmessung neuer Werkstoffe . . . . . . . . 114

4. Methoden des Bilanzaufbaus für den Werkstoffverbrauch . . . . . . 130

Viertes Kapitel

Die Hauptentwicklungstendenzen und die Ökonomik der Produktion neuer Werkstoffe . . . . . . . . . . . . . . . . . 143

1. Dic Strukturverschiebungen in der Produktion neuer Werkstoffe . . 143

2. Die Entwicklung des Volumens und der Struktur der Produktion von Plasten und daraus gefertigten Erzeugnissen . . . . . . . . . . . .

3. Technisch-ökonomische Charakteristika der traditionellen und neuen Werkstoffe ................... 161 


\section{Ftinftes Kapitel}

Progressive Strukturverschiebungen und die Effektivität des Verbrauchs neuer Werkstoffe . . . . . . . . . . . . . . . . . . 180

1. Allgemeine Tendenzen des Werkstoffverbrauchs ......... 180

2. Die Hauptrichtungen des Plastverbrauchs . . . . . . . . . . . 196

3. Die Verwendung von Sekundärrohstoffen . . . . . . . . . . 216

4. Die Effektivität des Einsatzes von Plast in der Volkswirtschaft der UdSSR . . . . . . . . . . . . . . . . . . 219

\section{Sechstes Kapitel}

Okonomische Analyse des Verbrauchs neuer Werkstoffe und Materialien in den wichtigsten $\mathrm{Zweigen}$ und Objekten . . . . . . . . . . . 235

1. Konstruktionswerkstoffe im Maschinenbau . . . . . . . . 235

2. Elektroisolierstoffe . . . . . . . . . . . . . 247

3. Werkstoffe auf der Basis der chemischen und chemisch-mechanischen Holzverarbeitung . . . . . . . . . . . . . . . . 261

4. Werkstoffe und Materialien für Verpackungs- und Umhüllungsmittel . . 275

5. Werkstoffe und Materialien für die Leichtindustrie . . . . . . . 292

6. Baustoffe ..................... 301

Personenregister ................... . 317

Sachregister . . . . . . . . . . . . . . . 319 